OPEN ACCESS

Edited by:

Feng $L u$,

Yangzhou University, China

Reviewed by:

Jianbing $\mathrm{Mu}$

National Institutes of Health (NIH),

United States

Istvan Albert,

Pennsylvania State University, United States

*Correspondence:

Cizhong Jiang

czjiang@tongji.edu.cn

Qingfeng Zhang

qfzhang@tongji.edu.cn

qfzhangsh@aliyun.com

tThese authors have contributed

equally to this work

Specialty section:

This article was submitted to

Molecular Medicine,

a section of the journal

Frontiers in Cell and Developmental

Biology

Received: 09 September 2019

Accepted: 18 October 2019

Published: 01 November 2019

Citation:

Liu M, Lu B, Fan Y, He X, Shen S, Jiang $C$ and Zhang Q (2019) TRIBE Uncovers the Role of Dis3 in Shaping the Dynamic Transcriptome in Malaria Parasites. Front. Cell Dev. Biol. 7:264. doi: $10.3389 /$ fcell.2019.00264

\section{TRIBE Uncovers the Role of Dis3 in Shaping the Dynamic Transcriptome in Malaria Parasites}

\author{
Meng Liu't ${ }^{1+}$ Binbin $\mathrm{Lu}^{2 \dagger}$, Yanting Fan ${ }^{2}$, Xiaohui He${ }^{2}$, Shijun Shen ${ }^{1}$, Cizhong Jiang ${ }^{1,3 *}$ and \\ Qingfeng Zhang ${ }^{2 *}$
}

\begin{abstract}
1 Institute of Translational Research, Tongji Hospital, Shanghai Key Laboratory of Signaling and Disease Research, The School of Life Sciences and Technology, Tongji University, Shanghai, China, ${ }^{2}$ Research Center for Translational Medicine, Key Laboratory of Arrhythmias of the Ministry of Education of China, East Hospital, Tongji University School of Medicine, Shanghai, China, ${ }^{3}$ The Research Center of Stem Cells and Ageing, Tsingtao Advanced Research Institute, Tongji University, Shanghai, China
\end{abstract}

Identification of RNA targets of RNA-binding proteins (RBPs) is essential for complete understanding of their biological functions. However, it is still a challenge to identify the biologically relevant targets of RBPs through in vitro strategies of RIP-seq, HITS-CLIP, or GoldCLIP due to the potentially high background and complicated manipulation. In malaria parasites, RIP-seq and gene disruption are the few tools available currently for identification of RBP targets. Here, we have adopted the TRIBE (Targets of RNA binding proteins identified by editing) system to in vivo identify the RNA targets of PfDis3, a key exoribonuclease subunit of RNA exosome in Plasmodium falciparum. We generated a transgenic parasite line of PfDis3-ADARcd, which catalyzes an adenosine (A)-toinosine (I) conversion at the potential interacting sites of PfDis3-targeting RNAs. Most of PfDis3 target genes contain one edit site. The majority of the edit sites detected by PfDis3-TRIBE locate in exons and spread across the entire coding regions. The nucleotides adjacent to the edit sites contain $\sim 75 \%$ of $A+T$. PfDis3-TRIBE target genes are biases toward higher RIP enrichment, suggesting that PfDis3-TRIBE preferentially detects stronger PfDis3 RIP targets. Collectively, PfDis3-TRIBE is a favorable tool to identify in vivo target genes of RBP with high efficiency and reproducibility. Additionally, the PfDis3-targeting genes are involved in stage-related biological processes during the blood-stage development. Thus PfDis3 appears to shape the dynamic transcriptional transcriptome of malaria parasites through post-transcriptional degradation of a variety of unwanted transcripts from both strands in the asexual blood stage.

Keywords: malaria, Plasmodium falciparum, TRIBE, Dis3, RIP-seq

\section{INTRODUCTION}

Plasmodium falciparum, a unicellular apicomplexan parasite, causes the most severe clinical outcome of malaria in human. To date, malaria remains a major global health threat with an estimated 400,000 malaria deaths each year worldwide (World Health Organization [WHO], 2018). The pathogenesis of $P$. falciparum in human results from the intra-erythrocytic developmental cycle (IDC), and each step of which is controlled by a precisely timed cascade of gene 
expression. Throughout the 48-h IDC, a majority of mRNAs reach peak abundance at only one time point, suggesting a strong correlation between transcriptome regulation and pathogenesis (Bozdech et al., 2003).

Recent years, post-transcriptional regulation has emerged as an important pathway in orchestrating biological processes on a transcriptome-wide scale throughout the IDC (Rai et al., 2014; Vembar et al., 2016). Nascent RNA sequencing revealed the pervasive distribution of nascent transcripts in the genome of this parasite, supporting the existence of an overlooked posttranscriptional regulation pathway in shaping the steady-state transcriptome in P. falciparum (Lu et al., 2017; Painter et al., 2018). For instance, by an inducible gene knockout strategy, the RNA exosome complex-associated $3^{\prime}-5^{\prime}$ exoribonuclease subunit, PfDis3, was found to degrade different kinds of antisense lncRANs and a few mRNAs (Droll et al., 2018). Moreover, PfRNase II, an ortholog of Dis3, has been reported to silence a subgroup of the primary virulence genes, var, by degrading nascent mRNA in situ (Zhang et al., 2014). These studies point to a critical regulatory function of RNA exosome in shaping the transcriptome of malaria parasites by surveillance of various transcripts in the life cycle. However, due to the failure to generate and isolate the pure cells of DiCre recombinasemediated conditional PfDis3 knockout line, the exact targets and related biological role of PfDis 3 in regulating transcriptome of malaria parasites remain to be clarified by other approaches.

Conventional methods to identify in vivo targets of RNAbinding proteins (RBP) include CLIP (crosslinking and immunoprecipitation) and variants thereof (Ule et al., 2003, 2005; Corden, 2010; Moore et al., 2014) and RIP (RNA immunoprecipitation) (Gilbert and Svejstrup, 2006). These methods are based on immunoprecipitation with specific antibodies recognizing the RBPs. After covalently binding of RBP to its targets, unprotected RNAs are digested and the remaining RBP-bound RNAs are isolated for high throughput sequencing. These approaches need a high-affinity and specific antibody. The low efficiency of crosslinking step $(\sim 1-5 \%)$ in CLIP also limits the yield of real targets in IP experiments (Darnell, 2010). It therefore requires large amounts of starting materials (almost millions of cells) and may raise the problem of high false-positive rate which is usually observed in IP experiments. In P. falciparum, RIP assay is still the main method to identify targets of RBPs since the CLIP-derived techniques such as PAR-CLIP or GoldCLIP are still not established in this organism so far. To overcome the defects of IP-based methods, McMahon et al. has developed a novel technique termed TRIBE (targets of RNA binding proteins identified by editing) to identify the RNA substrates of RBPs in vivo. This system fuses a catalytic domain of the RNA-editing enzyme ADAR (ADARcd) to a RBP of interest and expresses the fusion protein in vivo (McMahon et al., 2016). ADAR consists of two double-stranded RNA-binding domains (dsRBDs) and a catalytic domain (ADARcd) that deaminates adenosine to inosine (Bass and Weintraub, 1998; Keegan et al., 2004). By coupling the RBP to only ADARcd, the RBP targets are marked with de novo editing events which are identified by RNA sequencing (McMahon et al., 2016). Compared to the methods mentioned above, no immunoprecipitation is needed in TRIBE. Thus, problems like low efficiency of crosslinking and requirement of high affinity, specific antibody or terminal tagging of RBP of interest can be avoided (McMahon et al., 2016). Moreover, in TRIBE assay RNA is simply extracted from cells and sequenced by routine RNA-seq assay. Thus, it requires much less cells than RIP-seq. More importantly, it provides a standard but practice-friendly protocol compared with that of CLIPs (McMahon et al., 2016).

In this study, we sought to adopt the TRIBE technique in $P$. falciparum, by which we were able to identify the substrates of PfDis3 in vivo. We have generated Pfdis3-adar transgenic parasite line by CRISPR-Cas9 gene editing system, and used it to identify PfDis3-targeted transcripts by TRIBE throughout the IDC in P. falciparum. Through detecting de nova editing events catalyzed by the PfDis3-ADARcd fusion protein, we found that the majority of the editing sites were located in exonic regions. For the 5602 protein-coding genes in the genome of 3D7 strain, we have identified 2032, 2061 and 2303 genes with editing signals on the sense transcripts, whereas 1522, 2119 and 2187 on the antisense transcripts at ring, trophozoite and schizont, respectively. The TRIBE results were further supported by RIP-seq assay with Pfdis3-tag transgenic line by comparative analysis. Moreover, our target genes of PfDis3 were validated by inducible Pfdis3 gene knockdown analysis. Taken together, by development of TRIBE technique in $P$. falciparum, we reveal that PfDis3 targets are enriched in genes involved in multiple biological processes that are highly relevant to their respective time points of development in IDC, indicating a fundamental function of PfDis3 in surveillance of gene expression throughout the asexual stage in malaria parasites.

\section{RESULTS}

\section{Generation of Pfdis3-Adar Transgenic Parasite Line by CRISPR-Cas9}

To avoid side effect or growth defect by overexpression of PfDis3-ADARcd, we decided to generate an endogenous integration of the catalytic domain of ADAR enzyme from Drosophila into Pfdis3 gene locus (Figures 1A,B). In order to use CRISPR/Cas9 gene editing technique to achieve fast endogenous integration of the catalytic domain of ADAR enzyme, we constructed the plasmid pL6-PfDis3-Ty1-ADARcd targeting the C-terminus of Pfdis 3 gene and co-transfected with Cas9 expression vector pUF1-cas9 into 3D7 strain (Figure 1B). Approximately 3 weeks after transfection, parasites carrying pL6PfDis3-Ty1-ADARcd/pUF1-cas9 was obtained by drug selection with both WR99210 (WR) and blasticidin S deaminase (BSD). Next, we cloned this transgenic parasite line and confirmed the integration of Ty1-ADARcd at C-terminus of Pfdis3 gene by PCR (Figure 1C). Western blot assay further confirmed the expression of transgenic ADARcd with the expected molecular weight (MW) (Figure 1D). The specific recognition of the PfDis3-HATy1 fusion protein by antibodies was critical for reducing the background of RIP-seq assay.

TRIBE technology theoretically can detect adenosine (A)-to-inosine (I) editing events on the target transcripts of 
A

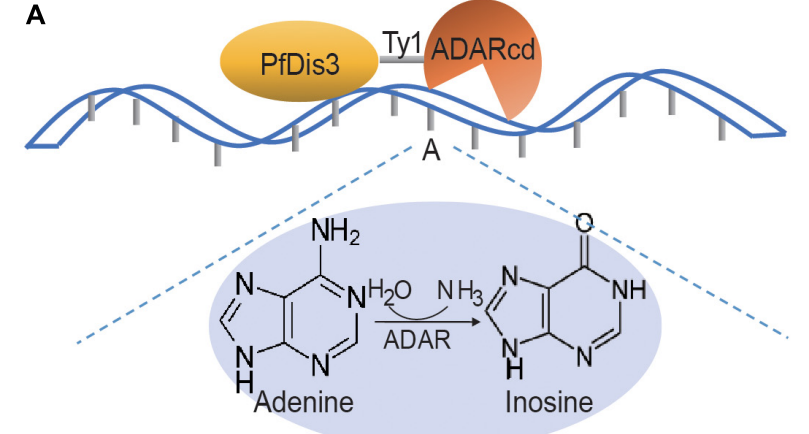

C
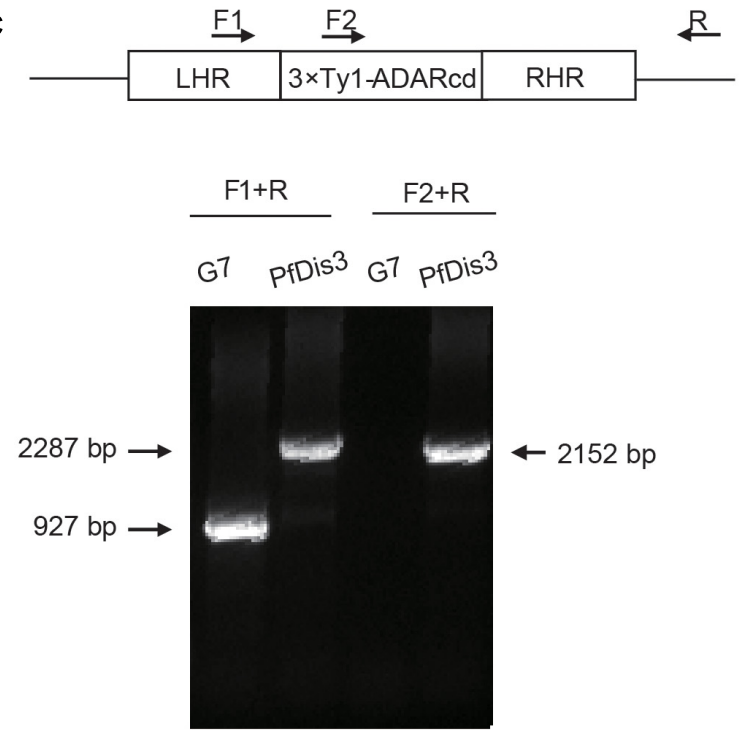

B

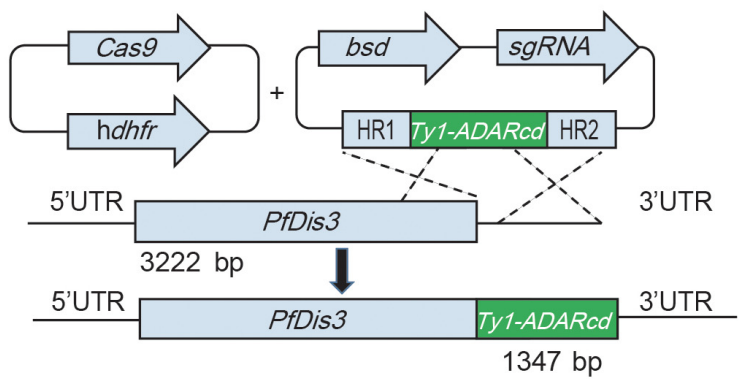

D

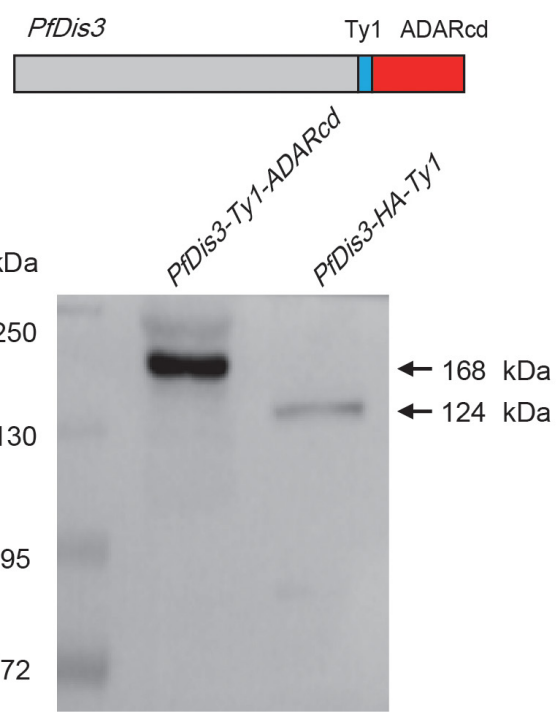

FIGURE 1 | Generation of Pfdis3-adar transgenic parasite line by CRISPR-Cas9. (A) PfDis3-ADARcd fusion protein was constructed by attaching a catalytic ADAR protein (ADARcd) to PfDis3. The ADARcd possess a deaminase domain that catalyzes an adenosine-to-inosine conversion. And the editing specificity of the fusion protein is determined by the RNA recognition features of PfDis3. (B) Schematic diagram showing the generation of PfDis3-ADARcd transfectant lines by the CRISPR-Cas9 system. The plasmid carrying a single guide RNA (sgRNA) and the Cas9 endonuclease were co-transfected. bsd, blasticidin S deaminase. hdhfr, human dihydrofolate reductase. HR, homology region. (C) GPCR analysis of PfDis3-ADARcd lines. LHR, homology region upstream of Pfdis3-Ty1-ADARcd. RHR, homology region downstream of PfDis3-Ty1-ADARcd. F1, forward primer 1 (5' gaattgttccatttaagcttttatg $\left.3^{\prime}\right)$. F2, forward primer 2 (5' cagaagtacatactaaccaagatc $\left.3^{\prime}\right)$. R, reverse primer ( $5^{\prime}$ acaatgttgttaaattaagtattatg $\left.3^{\prime}\right)$. (D) Western blot analysis of PfDis3-ADARcd lines with antibody against Ty1 epitope.

PfDis3 since the PfDis3-ADARcd protein doesn't contain the RNA recognition domain of ADAR (Figure 1A). To eliminate the background due to genomic mutation or Single Nucleotide Polymorphism (SNPs), we have re-sequenced the genome sequence of wild-type 3D7-G7 clone for transfection of PfDis3-ADARcd construct. Next, by strand-specific RNA sequencing of PfDis3-ADARcd transgenic parasite clone, we were able to identify the de novo editing events on the target transcripts of PfDis3 on individual strands of chromosomes. The wild-type 3D7-G7 clone was used as control to exclude endogenous RNA editing events in the parasites. To comprehensively identify the targets of Pfdis3, we harvested the synchronized parasites at ring (R), trophozoite (T) and schizont (S) asexual developmental stages, respectively. Meanwhile, the PfDis3-HA-Tyl tagging line was collected in parallel for RIP-seq analysis with specific antibody against Tyl epitope.

\section{PfDis3-ADARcd Edits Evenly Along the Transcripts From 5'UTR to 3'UTR}

By nucleotide sequence-based stranded comparative transcriptome analysis between PfDis3-ADARcd and WT 3D7-G7 clone, a total of 3643,3626, 3387 editing sites on the sense transcripts and 2671,3765, 3789 editing sites on the antisense transcripts were detected for the three asexual developmental stages (R, T, S), respectively. These editing sites correspond to 2032, 2061, 2303 genes and 1522, 2119, 2187 antisense non-coding RNAs, respectively. Besides the known structured RNAs processed by PfDis3 protein such as rRNAs and 
small nucleolar RNAs, we also identified many PfDis3 targets of various functions corresponding to their respective developing stages (Supplementary Table S1).

The majority of the de novo edit sites of PfDis3-ADARcd locate in exons (Figure 2A). Moreover, the edit sites are evenly distributed along the transcripts with minor enrichment in the 3'UTR (Figures 2B,C). This enrichment feature is consistent with Dis3's catalytic feature as endonuclease and exonuclease (Lebreton et al., 2008; Schaeffer et al., 2009; Schneider et al., 2009). The number of the edit sites in each transcript ranges from 1 to 15. Most of transcripts contain single edit site (Figure 2D). Interestingly, the editing events are highly reproducible both in position and efficiency (Figure 2D, inset and Supplementary Figure S1A). The median percentage editing levels of de novo

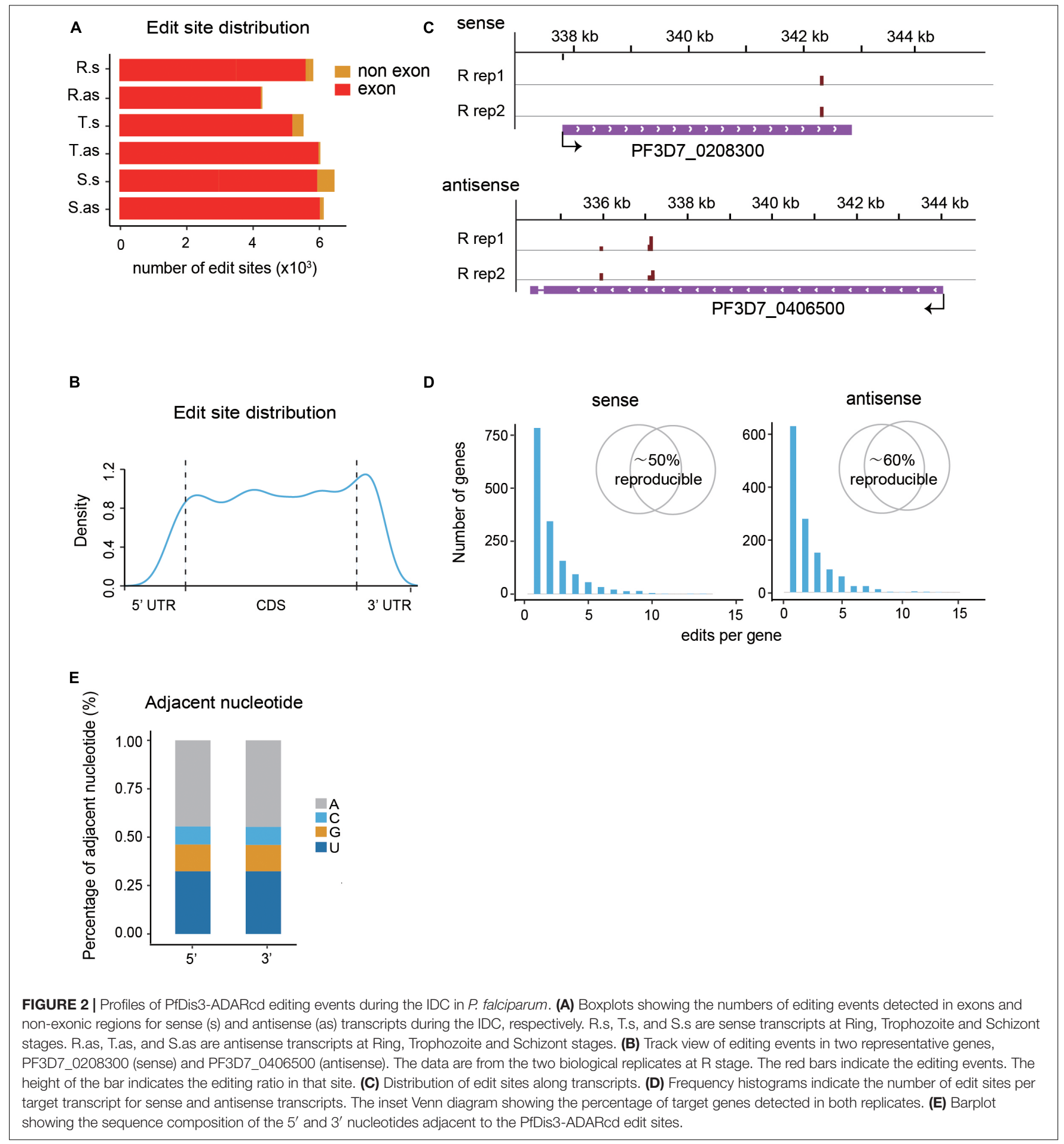


editing events in both sense and antisense transcripts during the IDC are about $8 \%$ (Supplementary Figure S1B). To explore whether the number of editing sites in each gene would infect editing ratio, we grouped transcripts by their total number of editing sites and compared their editing ratio among different groups. The results show no obvious correlation between the number of editing sites and editing ratio for both sense and antisense transcripts (Supplementary Figure S1C).

It has been reported previously that the $P$. falciparum genome has the highest AT composition among all the organisms sequenced to date (Gardner et al., 2002). Consequently, the mRNA transcriptome possesses higher level of adenosine in $P$. falciparum than other organisms. We wondered whether there is sequence composition bias in flanking sequences of the editing sites. To this end, we examined the adjacent nucleotides of editing sites. Unlike the former study that ADAR preferred an editing sequence of UAG (Rahman et al., 2018), the results show that the most frequent adjacent nucleotide is A, the second $T$, the third $G$, then $C$ (Figure 2E). The special editing environment of PfDis3-TRIBE may reflect the unique composition of $P$. falciparum genome.

\section{TRIBE Exhibits Higher Sensitivity and Reproducibility in Identification of PfDis3 Targets in P. falciparum}

To further assess the reliability of our editing result, we performed a series of strand-specific RIP-seq experiments with PfDis3-HA-Ty1 parasite line to identify the potential targets of PfDis3. The RIP enrichment ratio relative to the expression level of transcripts was calculated and normalized. We observed a high correlation of RIP signals in the biological replicates (Supplementary Figure S2). The targets identified by RIP are also reproducible (Supplementary Figure S2, Venn diagram). Due to the low resolution, RIP signals are broad and spread across the gene body (Figures 3A,B). Interestingly, TRIBE detected editing sites in the genes with RIP signals in the gene body. Notably, TRIBE detected editing sites with 1-bp resolution. Surprisingly, the target genes identified by TRIBE are biased toward higher RIP signals when compared to the target genes identified by RIP (Figure 3C). Indeed, 36, 39, and 47\% of RIP targets are also TRIBE targets at R, T and S stages, respectively (Figure 3C, Venn plot). Consistently, it has been reported previously that TRIBE was able to detect high-confidence CLIP targets (McMahon et al., 2016). Moreover, the correlation coefficient between replicates in TRIBE assay is higher and has a much smaller variation than that of RIP assay for either sense or antisense targets (Figure 3D and Supplementary Figures S1A, S2). Collectively, these findings suggest that TRIBE can reliably identify the targets of PfDis3 with high resolution in $P$. falciparum.

\section{PfDis3 Regulates the Dynamical Transcriptional Program During the Asexual Blood Stage in P. falciparum}

We next investigated the dynamics of PfDis3 targets during the IDC. The results show that there are no editing sites in $\sim$ one third of genes across the IDC whereas one sixth of genes persistently contain editing sites. The rest of transcripts dynamically contain editing sites (Figure 4A). The common targets of PfDis 3 across the IDC account for 41,40 , and $36 \%$ of sense targets and 52, 37, and 36\% of antisense targets for R, T and $\mathrm{S}$ stages, respectively. In contrast, only 27,19 , and $28 \%$ of sense targets and 15,21 , and $28 \%$ of antisense targets are specific to R, $\mathrm{T}$ and $\mathrm{S}$ stages, respectively (Figure $4 \mathrm{~B}$ ). This indicates that the targets of PfDis 3 only show stage specificity to a certain extent. We further examined the correlation between the sense targets and antisense targets of PfDis 3 at $\mathrm{R}, \mathrm{T}$ and $\mathrm{S}$ stages, respectively. The results show that 26,38 , and $42 \%$ of sense targets contain edit site(s) in the corresponding antisense targets for R, T and S stages, respectively. Similarly, 35, 37, and $44 \%$ of antisense targets contain edit site(s) in the corresponding sense targets for $\mathrm{R}, \mathrm{T}$ and $\mathrm{S}$ stages, respectively (Figure 4C).

To understand the functions of the target genes of PfDis3, we performed Gene Ontology (GO) analysis of the target genes. The results show that the target genes at $\mathrm{R}, \mathrm{T}$ and $\mathrm{S}$ stages are enriched in the stage-related functions (Figures 5A,B and Supplementary Figures S3A,B). For example, the sense target genes at $\mathrm{R}$ stage are enriched for entry into host cell, pathogenesis, invasion, etc. (Figure 5A). This is consistent with infection and inhabitation of the parasites in red cells at $\mathrm{R}$ stage. In contrast, the antisense target genes at $\mathrm{T}$ stage are enriched for DNA replication, protein folding, metabolic process, etc. (Figure 5B). This is consistent with proliferation of the parasites at $\mathrm{T}$ stage.

We next constructed conditional Pfdis3 knock down (KD) parasite lines PfDis3-DD with ribozyme system (Droll et al., 2018) to investigate how PfDis3 impacts gene transcription. We used RNA-seq to measure gene expression levels that are highly reproducible (Supplementary Figure S3C). After Pfdis3 knockdown by drug induction, a number of genes are significantly differentially expressed. Especially, there are much more significantly up-regulated genes than down-regulated genes at $\mathrm{R}$ and $\mathrm{T}$ stages (Figure 5C). Intriguingly, the majority of genes in the GO term "entry into host cell" are up-regulated at R stage upon Pfdis3 knockdown. Similarly, the majority of genes in the GO term "DNA replication" are up-regulated at T stage upon Pfdis3 knockdown (Figure 5D). This is consistent with the functions of the target genes (Figures 5A,B).

\section{DISCUSSION}

The RNA exosome complex is highly conserved in eukaryotic organisms. In P. falciparum, the RNA exosome is composed of seven distinct core exosome subunits and two canonical $3^{\prime}-5^{\prime}$ exoribonuclease factors: PfDis3 and PfRrp6 (Droll et al., 2018). For Dis3 protein, it harbors both processive exonucleolytic and endonucleolytic activities originating from the RNB and PIN domains, respectively (Lebreton et al., 2008; Schaeffer et al., 2009; Schneider et al., 2009). In yeast, Dis3 is present in both the nucleus and cytoplasm (Gudipati et al., 2012; Schneider et al., 2012) and human Dis3 proteins are differently localized (Staals et al., 2010; Tomecki et al., 2010; Lubas et al., 2013). Disruption of human dis3 (hRrp44) gene uncovered the cryptic transcription upstream of active human promoters (Preker et al., 2008). By 

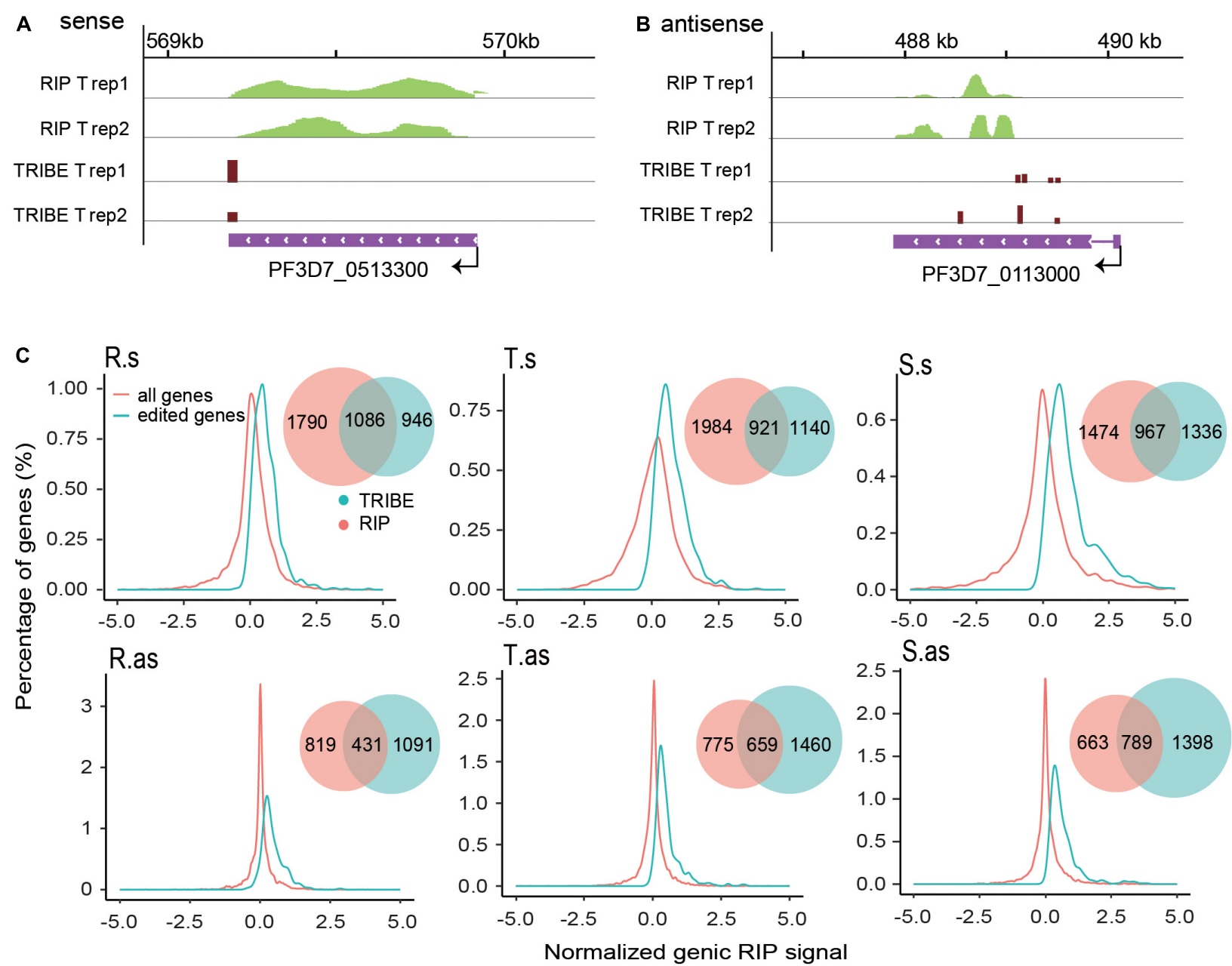

D
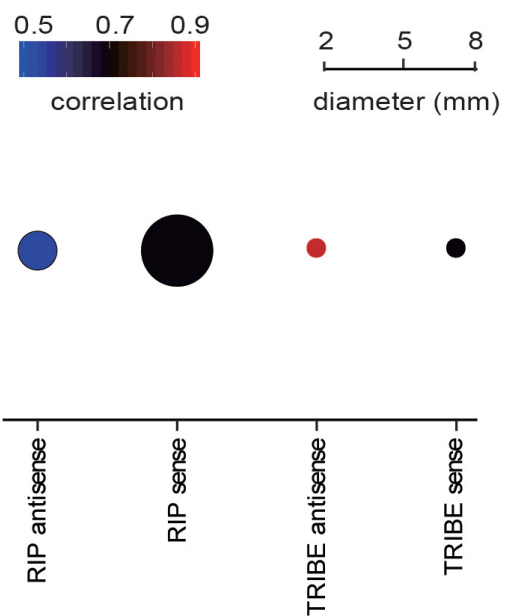

FIGURE 3 | PfDis3-TRIBE identifies targets with higher sensitivity and reproducibility than PfDis3-RIP in P. falciparum. (A,B) Track view showing RIP signals and editing events in the two representative target genes, PF3D7_0513300 (a, sense) and PF3D7_0113000 (b, antisense). The data are from the two biological replicates of RIP and TRIBE, respectively. (C) Distribution of normalized genic RIP signal in all RIP target genes (red) and PfDis3-ADARcd edited genes (green). The inset Venn plot shows the overlap of the target genes identified by both PfDis3-TRIBE (green) and PfDis3-RIP (red). R.s, T.s, and S.s are sense transcripts at Ring, Trophozoite and Schizont stages. R.as, T.as, and S.as are antisense transcripts at Ring, Trophozoite and Schizont stages. (D) Bubble plot showing the Pearson correlations between the replicates for PfDis3-TRIBE and PfDis3-RIP at ring (R), trophozoite (T) and schizont (S) stages, respectively. The color indicates the mean value of correlation coefficients. The size of the circle indicates the variance of correlation coefficients. 
A

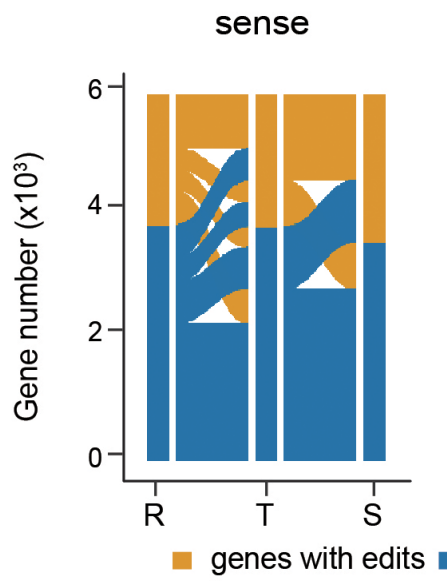

antisense

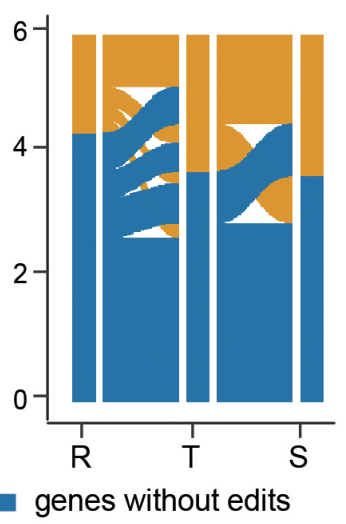

B

sense

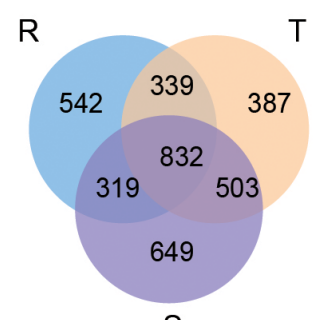

$\mathrm{S}$ antisense

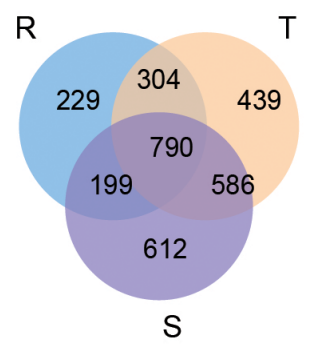

\section{C $\mathrm{R}$}

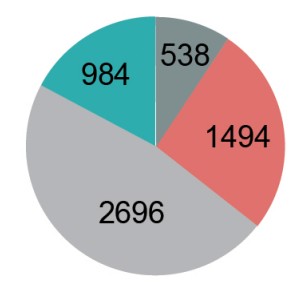

antisense only
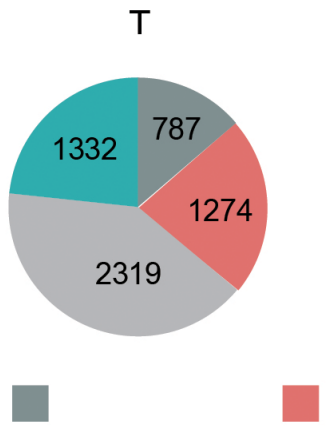

sense only
$S$

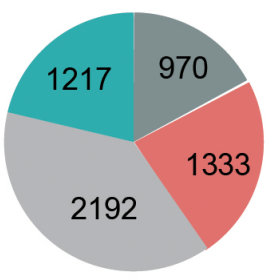

genes without edits

FIGURE 4 | Dynamics and features of PfDis3-TRIBE target genes during the IDC in P. falciparum. (A) Dynamic change of editing events in sense transcripts and antisense transcripts during the IDC. R, ring. T, trophozoite. S, schizont. (B) Overlap of PfDis3-TRIBE sense and antisense target transcripts at ring (R), trophozoite (T) and schizont (S) stages. (C) Pie chart showing the number of categorized target genes detected by TRIBE at ring (R), trophozoite (T) and schizont (S) stages, respectively. sense only, Pfdis3 binds to the sense transcripts but not the antisense transcripts of the genes. antisense only, Pfdis 3 binds to the antisense transcripts but not the sense transcripts of the genes. sense and antisense, Pfdis3 binds to both antisense and sense transcripts of the genes. Genes without edits, genes with no editing events detected.

combining the transcriptomic analysis of human cell with dis3 gene mutation and Photoactivatable Ribonucleoside-Enhanced Cross-Linking and Immunoprecipitation (PAR-CLIP) assay, the RNA polymerase II transcriptome in humans was found to be controlled by Dis3 through decay of unwanted transcripts associated with $\sim 50 \%$ of transcribed protein-coding genes and unannotated genomic regions covering $\sim 70 \%$ of the genome. In P. falciprum, PfDis3 was detected predominately in the cytoplasmic fraction during asexual blood stage, but the majority of target transcripts of PfDis3 were antisense lncRNAs measured by DiCre knockout and RNA-seq (Droll et al., 2018). Here, by using TRIBE analysis, we uncover that PfDis3 is a global post-transcriptional regulator of protein coding and noncoding transcripts over the course of blood-stage development of the parasites. The function of PfDis3 in shaping cellular transcriptome is likely evolutionary conserved in eukaryotes from Plasmodium to human.

The human malaria parasite $P$. falciparum has a special nucleotide composition of genome with extremely higher AT content compared to other organisms (Gardner et al., 2002).
Moreover, the mRNA transcriptome displays even stronger adenosines bias of about 45\% (Baumgarten et al., 2019). The special sequence composition and other reasons make it hard to identify RBP targets in $P$. falciparum with traditional immunoprecipitation-based methods. In CLIP, crosslinking needs to be performed beforehand, raising a bias of preferential crosslinking of uredines (Fecko et al., 2007). It has not been reported any successful CLIP experiment in $P$. falciparum to date. RIP also faces problems like choice of RNase and the fragmentation condition that has a significant impact on the detected targets (Lambert et al., 2014). Many RIP results are not reproducible due to the non-specific binding of antibody in P. falciparum. To circumvent these issues, we adopted PfDis3TRIBE in $P$. falciparum to identify Pfdis3 targets throughout IDC for the first time. We first identified Pfdis3 targeted sense/antisense transcripts at ring, trophozoite and schizont stage, respectively (Supplementary Table S1). We found that many Pfdis3 targets were antisense transcripts. Both sense and antisense Pfdis3 targets were enriched in biological processes that are highly relevant for their developmental stages, indicating 
A

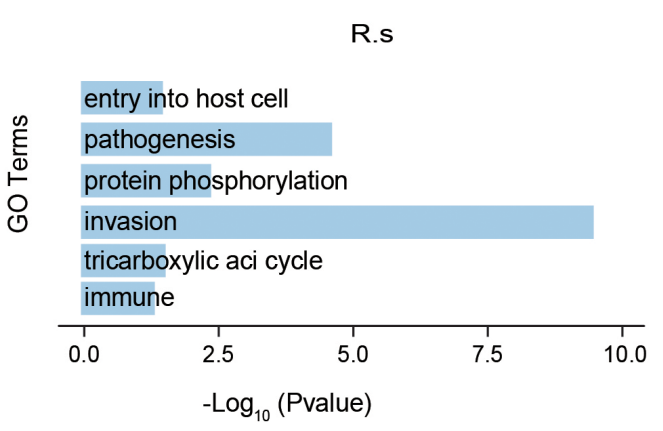

B

T.as

DNA replication

environment response

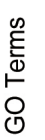

protein folding

metabolic process

DNA/RNA

ER to Golgi vesicle-mediated transport pentose-phosphate shunt

microtubule nucleation
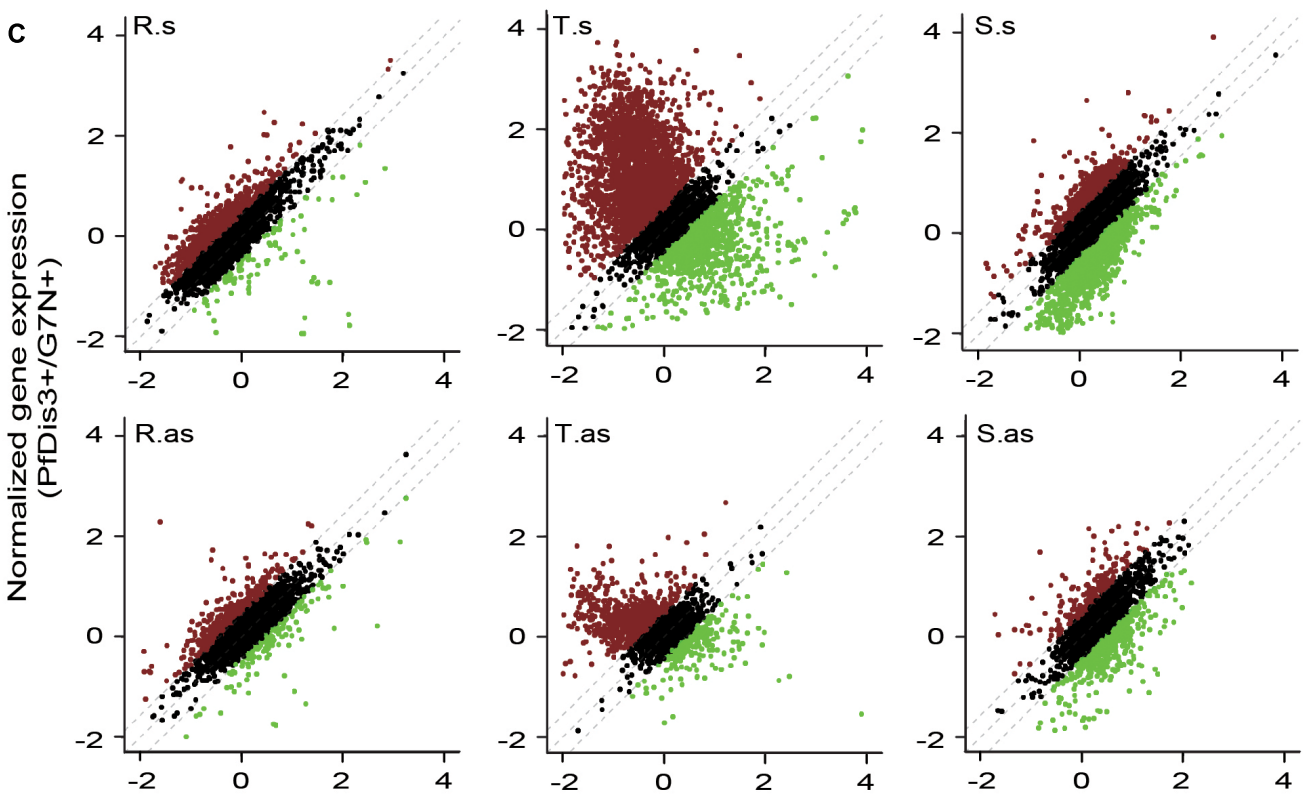

- up regulate

- non DEGs

- down regulate
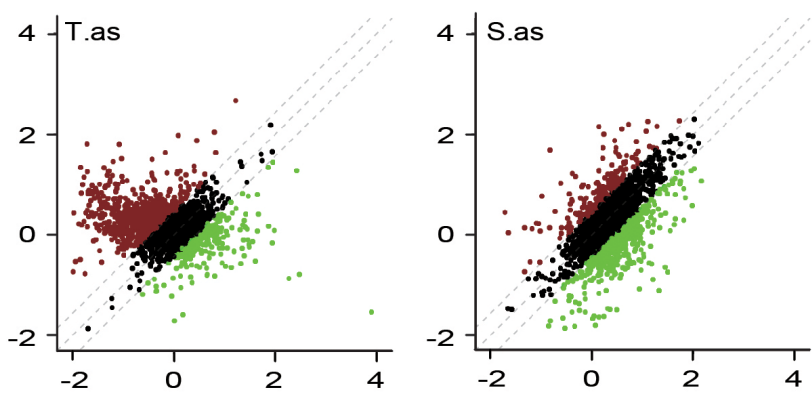

Normalized gene expression (PfDis3-/G7N-)

D

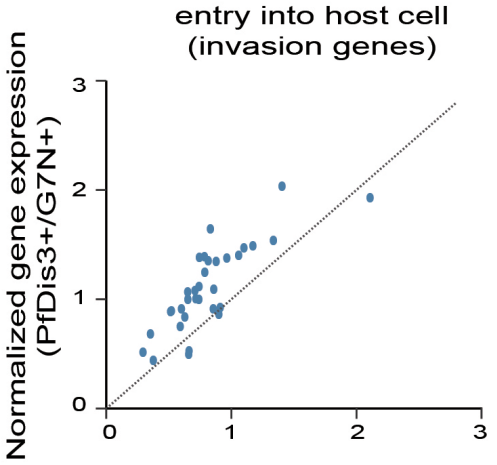

DNA replication

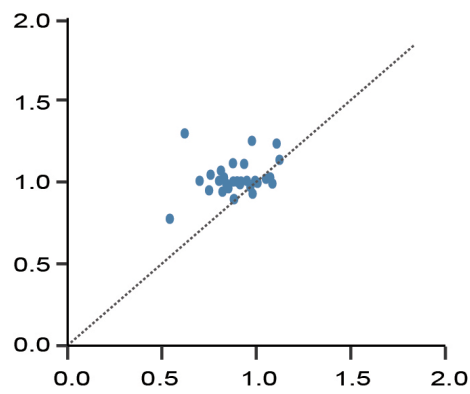

Normalized gene expression

(PfDis3-/G7N-)

FIGURE 5 | The functions of PfDis3-TRIBE target genes during the IDC in P. falciparum. (A) Enriched Gene Ontology (biological processes) terms for PfDis3-TRIBE sense target genes at ring stage. (B) Enriched GO terms for PfDis3-TRIBE antisense target genes at trophozoite stage. (C) Scatter plots showing expression levels of sense (top) and antisense (bottom) transcripts at R, T and $S$ stages with ( $Y$ axis) and without ( $X$ axis) Pfdis3 knock down. Transcripts with fold change $\geq 1.35$ are differentially expressed transcripts. (D) Scatter plots showing that the majority of the genes in the GO term "entry into host cell" are up-regulated at R stage upon Pfdis3 knockdown (left), and that the majority of the genes in the GO term "DNA replication" are up-regulated at T stage upon Pfdis3 knockdown (right). 
that Pfdis3 dynamically regulates the transcriptional program of $P$. falciparum throughout the IDC and maintains the steady state level of transcriptome.

We also performed RIP experiment and conditional Pfdis3 knockdown (KD) to evaluate the reliability of the target genes identified by PfDis3-TRIBE. PfDis3-TRIBE target genes were biased toward higher RIP enrichment, indicating that PfDis3-TRIBE preferentially detects stronger RIP-seq targets (Figure 3C). Moreover, most of the identified Pfdis 3 target genes across the IDC were upregulated upon Pfdis3 KD (Figure 5C). Taken together, all these results suggest that PfDis3-TRIBE is able to identify Pfdis3 targets with higher confidence and better reproducibility than RIP.

\section{MATERIALS AND METHODS}

\section{Plasmid Construction}

To generate plasmid Pfdis3-Ty1-ADARcd and Pfdis3-Ty1-ribo for transfection, we modified the plasmid $p L 6-g f p$ by replacing the $g f p$ box with a $\sim 1-k b$ homolog sequence flanking the $\mathrm{N}$ - or C-terminus of the target genes which contained three copies of Tylepitope and ADARcd or glmS ribozyme gene, and inserting a guide RNA sequence specific to the Pfdis3 gene (PF3D7_1359300) by In-Fusion PCR Cloning System, respectively (Supplementary Table S2). The resulting plasmids were pL6-Pfdis3-Ty1-ADARcd and pL6-Pfdis3-Ty1-Ribo. The plasmid pUF1-Cas9-infusion carrying Cas9 expression cassette was modified by replacing the original ydhodh gene with hdhfr.

\section{Parasite Culture and Transfection}

Plasmodium falciparum parasites were cultured in human red blood cell in culture medium (10.44 g/L RPMI-1640, $25 \mathrm{mM}$ HEPES, 10\% v/v Albumax I, $0.1 \mathrm{mM}$ hypoxanthine, $20 \mu \mathrm{g} / \mathrm{ml}$ gentamicin) under $5 \% \mathrm{O}_{2}, 3 \% \mathrm{CO}_{2}$ at $37^{\circ} \mathrm{C}$. For synchronization, ring-stage parasites were enriched with $5 \%$ sorbitol, late-stage parasites were enriched via 40 and $70 \%$ percoll. Fresh red blood cell were electroporated with $100 \mu \mathrm{g}$ of plasmid sgRNA and Cas9 in cytomix $\left(120 \mathrm{mM} \mathrm{KCl,} 2 \mathrm{mM}\right.$ EGTA, $10 \mathrm{mM} \mathrm{KH} \mathrm{PO}_{4}$, $25 \mathrm{mM}$ HEPES pH7.8, $0.15 \mathrm{mM} \mathrm{CaCl}_{2}, 5 \mathrm{mM} \mathrm{MgCl}_{2}$ ) and then the synchronization of late-stage parasites were added. After 2 or 3 cycles, transfected parasites were selected by WR99210 and blasticidin $\mathrm{S}$ deaminase drug. The integration DNA was validated by PCR following sequencing and the proteins were identified by Western blot.

\section{Western Blot}

Total proteins were extracted with $0.15 \%$ saponin, washed with cold PBS ( $\mathrm{pH}$ 7.4) until the supernatant was clear and then resuspended in $1 \times$ SDS-loading buffer following heating under $100^{\circ} \mathrm{C}$ for $5 \mathrm{~min}$. Proteins were separated by gel electrophoresis and transferred to a PVDF membrane. The membrane was blocked with $5 \%$ milk, incubated with primary antibody, washed with PBST, and then incubated with secondary antibody which could generate a chemiluminescent signal. The proteins were visualized by exposing to an imaging device. The materials used in this study were mouse anti-PfAldolase (1:1000, Abcam), mouse anti-Ty1 (1:500, Sigma), ECL Western blotting kit (GE healthcare) (Freeman, 2013).

\section{RNA Extraction and Stranded RNA-Seq}

Red blood cells infected by highly synchronous parasites were collected by centrifugation and resuspended in Trizol that could be stored in $-80^{\circ} \mathrm{C}$ for a long time. After centrifugation, the supernatant was saved and then RNA was extracted according to reagent specification of Direct-zol ${ }^{\mathrm{TM}}$ RNA MiniPrep (R2052). The integrality of RNA was validated by $2 \%$ agarose gel. Library construction was performed based on KAPA Stranded mRNASeq Kit (KK8421).

\section{RIP-Seq}

Infected red blood cells (iRBCs) were collected by centrifugation and resuspended in cold PBS (10 volume of iRBC). Parasites were extracted with $0.15 \%$ saponin and washed with cold PBS until the supernatant was clear. Lysis buffer $(50 \mathrm{mM}$ Tris-Cl pH7.4, $150 \mathrm{mM} \mathrm{NaCl}, 1 \mathrm{mM}$ EDTA, 1 mM EGTA, 1\% Triton $\mathrm{X}-100 / \mathrm{NP}-40)$ with protease inhibitors and RNase inhibitors was added to the parasite pellet $\left[1.5 \mathrm{ml} /\left(1 \times 10^{9}\right.\right.$ parasites $\left.)\right]$ and incubated with rotation for $1 \mathrm{~h}$ at $4^{\circ} \mathrm{C}$. Cell debris were spun out at $12000 \times g$ for $15 \mathrm{~min}$ at $4^{\circ} \mathrm{C}$ and supernatant was incubated with $10 \mu \mathrm{g}$ of anti-Tyl antibodies at $4^{\circ} \mathrm{C}$ for $3 \mathrm{~h}$ with rotation. For preparation of equilibrated protein-G magnetic beads, $50 \mu \mathrm{l}$ of protein-G magnetic beads were washed once with Wash Buffer (10 mM Tris-Cl pH 7.5, $150 \mathrm{mM} \mathrm{MgCl}_{2}$, $150 \mathrm{mM} \mathrm{KCl}, 0.1 \%$ Triton X-100), then washed once with Elution Buffer, and washed twice with Wash Buffer. Protein-G magnetic beads were mixed with supernatant with protease inhibitors and RNase inhibitors at $4^{\circ} \mathrm{C}$ overnight with rotation. Beads were washed twice with $500 \mu \mathrm{l}$ of Wash Buffer and once with PBS and then resuspended in $700 \mu \mathrm{l}$ of Trizol at $4^{\circ} \mathrm{C}$ for $10 \mathrm{~min}$. After removal of the beads, $140 \mu \mathrm{l}$ of chloroform was added to Trizol at room temperature for $5 \mathrm{~min}$ and mixture was centrifuged at $12000 \times g$ for $30 \mathrm{~min}$ at $4^{\circ} \mathrm{C}$. Supernatant was added to a new $1.5 \mathrm{ml}$ centrifuge tube and centrifuged at $12000 \times g$ for 2 min at $4^{\circ} \mathrm{C}$. Supernatant was mixed with $1 \mu \mathrm{l}$ of glycogen and equal volume of isopropanol on ice for $2 \mathrm{~h}$. Tube was centrifuged at $12000 \times g$ for $30 \mathrm{~min}$ at $4^{\circ} \mathrm{C}$. Pellet was washed twice with $1 \mathrm{ml}$ of $75 \%$ ( $\mathrm{vol} / \mathrm{vol}$ ) ethanol, then air-dried and resuspended in $20 \mu \mathrm{l}$ of RNase-free water (Chomczynski and Sacchi, 2006). DNase I was used to digest DNA for $15 \mathrm{~min}$ at room temperature. RNase-free water was added up to $100 \mu \mathrm{l}$, mixed with equal volume of chloroform/isoamyl alcohol $\mathrm{pH}<5.0$ (24:1), and then mixture was centrifuged at $12000 \times g$ for $5 \mathrm{~min}$. Supernatant was mixed with phenol/chloroform/isoamyl alcohol (25:24:1), and centrifuged at $12000 \times g$ for $5 \mathrm{~min}$. Supernatant was transferred to a new $1.5 \mathrm{ml}$ centrifuge tube with $1 \mu \mathrm{l}$ of glycogen, one-tenth volumes of $3 \mathrm{M}$ sodium acetate $(\mathrm{pH}$ 5.2 ), and 2.5 volumes of $100 \%$ ethanol and incubated at $80^{\circ} \mathrm{C}$ for $20 \mathrm{~min}$. Samples were centrifuged at $12000 \times g$ for $15 \mathrm{~min}$ and pellet was washed twice with $1 \mathrm{ml}$ of $75 \%$ ( $\mathrm{vol} / \mathrm{vol}$ ) ethanol, then air-dried and resuspended in $11 \mu \mathrm{l}$ of RNase-free water. Construction library was performed using KAPA Stranded mRNA-Seq Kit (KK8421). 


\section{RIP Sequencing Analysis}

RIP sequencing reads were trimmed with cutadapt (v11) by $10 \mathrm{bp}$ at each end. Reads with average quality score $\geq 20$ and length $\geq 50 \mathrm{bp}$ were retained. The strand specific reads were aligned with hisat2 (v2.1.0) (Kim et al., 2015) (parameters: rna-strandness RF -dta -no-discordant -no-mixed -no-unal). Read counts for sense/antisense transcripts were calculated using featureCounts (v1.6.1) with parameters: -M -p -B -C for all; -s 2 for sense transcripts; -s 1 for antisense transcripts (Liao et al., 2014). Both sense and antisense read counts were merged for library normalization between conditions. The final expression levels of sense/antisense transcripts were normalized as FPKM (Fragment Per Kilobase of transcript per Million reads mapped) for further analysis. RIP-seq (i.e., PfDis3-RIP and GFP-RIP) data were normalized with the transcript abundance of their corresponding time point. We also did quantile normalization for former normalized RIP data to make it comparable among samples. Only transcripts with minimum 1.2 fold of PfDis3RIP versus GFP-RIP were considered as PfDis3-RIP enriched transcripts. As biological replicates of the same treatment and developmental stage were highly correlated (Supplementary Figure S2), we combined them for the downstream analysis.

\section{Analysis of Sequencing Data for RNA Editing}

RNA editing data was analyzed as the previously published manual (McMahon et al., 2016) with modifications. In brief, pairend sequencing reads were trimmed with cutadapt (v11) by $10 \mathrm{bp}$ at each end, reads with average quality score $\geq 20$ and length $\geq 50$ bp were retained. Genomic DNA reads were aligned to the P. falciparum genome (plasmoDB.org, v3 release 32) (Gardner et al., 2002) using bwa (v 0.7.17-r1188) (Li and Durbin, 2009, 2010; Li, 2012) with default parameter. Strand specific RNA sequencing reads were aligned using Tophat2 (v2.1.1) (Kim et al., 2013) (parameters: -m 1 -g 2 -I 50000 -microexon-search no-coverage-search -library-type fr-firststrand). PCR duplicates were removed using sambamba (v0.6.6) (Tarasov et al., 2015) for editing analysis and reads were sorted using sambamba (v0.6.6). We next converted the sorted files to a matrix using a previously published perl script sam_to_matrix.pl (McMahon et al., 2016). Genomic nucleotide information and transcript nucleotide information were merged using bedtools (v2.27.1) (Quinlan and Hall, 2010) for further identification of edit sites.

Only sites with minimum 10 coverage and 5\% editing ratio were considered to be valid edit sites. gDNA coverage $\geq 30$ and uniformity of nucleotide were also required to avoid SNP. The editing ratios are highly reproducible for the biological replicates (Supplementary Figure S1A), therefore, we combined the biological replicates for the downstream analysis. RNA editing data was converted to bedgraph file format for display using R (v3.5.1).

\section{RNA-Seq Data Analysis}

Illumina adapters were removed with cutadapt (v11), reads with average quality score $\geq 20$ and length $\geq 50$ bp were retained. RNA sequencing reads were aligned using hisat2 (v2.1.0) (Kim et al., 2015) with strand specific mode (i.e., rna-strandness RF). Read counts for sense/antisense transcripts were obtained using featureCounts (v1.6.1) (Liao et al., 2014). Both sense and antisense read counts were merged for library normalization between conditions. FPKM of sense/antisense transcripts were calculated using R (v3.5.1). The gene expression levels were highly reproducible for the biological replicates of the same treatment and developmental stage (Supplementary Figure S3C), therefore, we used the replicate one for further analysis. Genes with minimum 1.35 fold (Pfdi3 KD versus G7) were considered as differentially expressed genes between two samples.

\section{Gene Ontology Analysis}

Gene ontology (GO) enrichment was performed using R (v3.5.1). GO terms database was downloaded from Plasmodb ${ }^{1}$. GO terms with $p$-value $\geq 0.05$ (Fisher exact test) and enriched gene number $\geq 5$ were considered to be enriched. We next classified our GO terms into different functional categories for better understanding.

\section{DATA AVAILABILITY STATEMENT}

The raw sequence data reported in this paper have been deposited in the Gene Expression Omnibus (GEO) under the accession number: GSE133654.

\section{AUTHOR CONTRIBUTIONS}

QZ and CJ conceived and designed the experiments. YF and $\mathrm{XH}$ generated transgenic parasite lines of PfDis3-ADARcd and PfDis3-DD. BL performed WB, TRIBE, and RNA-seq assay. ML and SS performed informatics analysis. QZ, CJ, and ML wrote the manuscript. All authors read and approved the final manuscript.

\section{FUNDING}

This work was supported by the National Natural Science Foundation of China (NSFC) (81630063 and 31671353), National Key R\&D Program of China Grant (2018YFA0507300) to QZ, and NSFC (31771419 and 31721003) to CJ.

\section{SUPPLEMENTARY MATERIAL}

The Supplementary Material for this article can be found online at: https://www.frontiersin.org/articles/10.3389/fcell.2019.00264/ full\#supplementary-material

FIGURE S1 | The PfDis3-ADARcd reproducibly edits certain sites in P.falciparum during the IDC. (A) The edit sites at sense (top) transcripts and antisense (bottom) transcripts are reproducible in editing frequency at ring (R), trophozoite $(T)$ and schizont (S) stages. Pearson correlation between biological replicates are shown. (B) Box plot showing the PfDis3-ADARcd editing levels in sense (s) transcripts and

${ }^{1}$ https://plasmodb.org/plasmo/ 
antisense (as) transcripts at ring (R), trophozoite ( $\mathrm{T}$ ) and schizont (S) stages. (C) Box plots showing the editing frequency of sense transcripts and antisense transcripts grouped by the number of edit sites. The middle line in the box indicates the mean of the editing frequency in the transcripts with more than one edit sites.

FIGURE S2 | Reproducibility of PfDis3-RIP assay across developmental stages. Correlation of genic RIP signals in sense (s) transcripts and antisense (as) transcripts between biological replicates. Pearson correlation coefficients between biological replicates are displayed at top left corner. The inset Venn diagram showing the overlap of PfDis3 targets identified by PfDis3-RIP between the two replicates. R, T, S indicates Ring, Trophozoite and Schizont stage, respectively.

\section{REFERENCES}

Bass, B. L., and Weintraub, H. (1998). An unwinding activity that covalently modifies its double-stranded RNA substrate. Cell 55:10.

Baumgarten, S., Bryant, J. M., Sinha, A., Reyser, T., Preiser, P. R., Dedon, P. C., et al. (2019). Transcriptome-wide dynamics of extensive m(6)A mRNA methylation during Plasmodium falciparum blood-stage development. Nat. Microbiol. doi: 10.1038/s41564-019-0521-7 [Epub ahead of print].

Bozdech, Z., Llinas, M., Pulliam, B. L., Wong, E. D., Zhu, J. C., and Derisi, J. L. (2003). The transcriptome of the intraerythrocytic developmental cycle of Plasmodium falciparum. PLoS Biol. 1:085. doi: 10.1371/journal.pbio.0000005

Chomczynski, P., and Sacchi, N. (2006). The single-step method of RNA isolation by acid guanidinium thiocyanate-phenol-chloroform extraction: twenty-something years on. Nat. Protoc. 1, 581-585. doi: 10.1038/nprot.2 006.83

Corden, J. L. (2010). Shining a new light on RNA-protein interactions. Chem. Biol. 17,316-318. doi: 10.1016/j.chembiol.2010.04.003

Darnell, R. B. (2010). HITS-CLIP: panoramic views of protein-RNA regulation in living cells. Wiley Interdiscip. Rev. RNA 1, 266-286. doi: 10.1002/wrna.31

Droll, D., Wei, G., Guo, G., Fan, Y., Baumgarten, S., Zhou, Y., et al. (2018). Disruption of the RNA exosome reveals the hidden face of the malaria parasite transcriptome. RNA Biol. 15, 1206-1214. doi: 10.1080/15476286.2018.1517014

Fecko, C. J., Munson, K. M., Saunders, A., Sun, G., Begley, T. P., Lis, J. T., et al. (2007). Comparison of femtosecond laser and continuous wave UV sources for protein-nucleic acid crosslinking. Photochem. Photobiol. 83:1394. doi: 10.1111/ j.1751-1097.2007.00179.x

Freeman, L. A. (2013). Western blots. Methods Mol. Biol. 1027, 369-385. doi: 10.1007/978-1-60327-369-5_18

Gardner, M. J., Hall, N., Fung, E., White, O., Berriman, M., Hyman, R. W., et al. (2002). Genome sequence of the human malaria parasite Plasmodium falciparum. Nature 419, 498-511.

Gilbert, C., and Svejstrup, J. Q. (2006). RNA immunoprecipitation for determining RNA-protein associations in vivo. Curr. Protoc. Mol. Biol. 27:11. doi: 10.1002/ 0471142727.mb2704s75

Gudipati, R. K., Xu, Z. Y., Lebreton, A., Seraphin, B., Steinmetz, L. M., Jacquier, A., et al. (2012). Extensive degradation of RNA precursors by the exosome in wild-type cells. Mol. Cell. 48, 409-421. doi: 10.1016/j.molcel.2012.08.018

Keegan, L. P., Leroy, A., Sproul, D., and O'connell, M. A. (2004). Adenosine deaminases acting on RNA (ADARs): RNA-editing enzymes. Genome Biol. 5:209.

Kim, D., Langmead, B., and Salzberg, S. L. (2015). HISAT: a fast spliced aligner with low memory requirements. Nat. Methods 12, 357-360. doi: 10.1038/nmeth. 3317

Kim, D., Pertea, G., Trapnell, C., Pimentel, H., Kelley, R., and Salzberg, S. L. (2013). TopHat2: accurate alignment of transcriptomes in the presence of insertions, deletions and gene fusions. Genome Biol. 14:R36. doi: 10.1186/gb-2013-144-r36

Lambert, N., Robertson, A., Jangi, M., Mcgeary, S., Sharp, P. A., and Burge, C. B. (2014). RNA Bind-n-Seq: quantitative assessment of the sequence and structural binding specificity of RNA binding proteins. Mol. Cell. 54, 887-900. doi: 10.1016/j.molcel.2014.04.016

Lebreton, A., Tomecki, R., Dziembowski, A., and Seraphin, B. (2008). Endonucleolytic RNA cleavage by a eukaryotic exosome. Nature 456, 993-996. doi: $10.1038 /$ nature 07480
FIGURE S3 | Functions of PfDis3-TRIBE target genes during the IDC in P. falciparum. (A) Enriched Gene Ontology (biological processes) terms for PfDis3 sense target transcripts at T (left panel) and S (right panel) stages, respectively. (B) Enriched GO terms for PfDis3 antisense target transcripts at ring R (left panel) and $S$ (right panel) stages, respectively. (C) Correlation for expression of sense (top) transcripts and antisense (bottom) transcripts between biological replicates. Pearson correlation coefficients between biological replicates are displayed at top left corner. R, T and S indicates Ring, Trophozoite, Schizont stage, respectively.

TABLE S1 | List of sense/antisense target genes identified by PfDis3-TRIBE during the IDC in $P$. falciparum.

TABLE S2 | Primers and homology sequences for our constructed parasites.

Li, H. (2012). Exploring single-sample SNP and INDEL calling with whole-genome de novo assembly. Bioinformatics 28, 1838-1844. doi: 10.1093/bioinformatics/ bts 280

Li, H., and Durbin, R. (2009). Fast and accurate short read alignment with burrowswheeler transform. Bioinformatics 25, 1754-1760. doi: 10.1093/bioinformatics/ btp324

Li, H., and Durbin, R. (2010). Fast and accurate long-read alignment with burrowswheeler transform. Bioinformatics 26, 589-595. doi: 10.1093/bioinformatics/ btp698

Liao, Y., Smyth, G. K., and Shi, W. (2014). featureCounts: an efficient general purpose program for assigning sequence reads to genomic features. Bioinformatics 30, 923-930. doi: 10.1093/bioinformatics/btt656

Lu, X. M., Batugedara, G., Lee, M., Prudhomme, J., Bunnik, E. M., and Le Roch, K. G. (2017). Nascent RNA sequencing reveals mechanisms of gene regulation in the human malaria parasite Plasmodium falciparum. Nucleic Acids Res. 45, 7825-7840. doi: 10.1093/nar/gkx464

Lubas, M., Damgaard, C. K., Tomecki, R., Cysewski, D., Jensen, T. H., and Dziembowski, A. (2013). Exonuclease hDIS3L2 specifies an exosomeindependent $3^{\prime}-5^{\prime}$ degradation pathway of human cytoplasmic mRNA. EMBO J. 32, 1855-1868. doi: 10.1038/emboj.2013.135

McMahon, A. C., Rahman, R., Jin, H., Shen, J. L., Fieldsend, A., Luo, W., et al. (2016). TRIBE: hijacking an RNA-editing enzyme to identify cell-specific targets of RNA-binding proteins. Cell 165, 742-753. doi: 10.1016/j.cell.2016.03.007

Moore, M. J., Zhang, C., Gantman, E. C., Mele, A., Darnell, J. C., and Darnell, R. B. (2014). Mapping argonaute and conventional RNA-binding protein interactions with RNA at single-nucleotide resolution using HITS-CLIP and CIMS analysis. Nat. Protoc. 9, 263-293. doi: 10.1038/nprot.2014.012

Painter, H. J., Chung, N. C., Sebastian, A., Albert, I., Storey, J. D., and Llinas, M. (2018). Genome-wide real-time in vivo transcriptional dynamics during Plasmodium falciparum blood-stage development. Nat. Commun. 9:2656. doi: 10.1038/s41467-018-04966-3

Preker, P., Nielsen, J., Kammler, S., Lykke-Andersen, S., Christensen, M. S., Mapendano, C. K., et al. (2008). RNA exosome depletion reveals transcription upstream of active human promoters. Science 322, 1851-1854. doi: 10.1126/ science. 1164096

Quinlan, A. R., and Hall, I. M. (2010). BEDTools: a flexible suite of utilities for comparing genomic features. Bioinformatics 26, 841-842. doi: 10.1093/ bioinformatics/btq033

Rahman, R., Xu, W., Jin, H., and Rosbash, M. (2018). Identification of RNAbinding protein targets with HyperTRIBE. Nat. Protoc. 13, 1829-1849. doi: 10.1038/s41596-018-0020-y

Rai, R., Zhu, L., Chen, H. F., Gupta, A. P., Sze, S. K., Zheng, J., et al. (2014). Genome-wide analysis in Plasmodium falciparum reveals early and late phases of RNA polymerase II occupancy during the infectious cycle. BMC Genomics 15:18. doi: 10.1186/1471-2164-15-959

Schaeffer, D., Tsanova, B., Barbas, A., Reis, F. P., Dastidar, E. G., SanchezRotunno, M., et al. (2009). The exosome contains domains with specific endoribonuclease, exoribonuclease and cytoplasmic mRNA decay activities. Nat. Struct. Mol. Biol. 16, 56-62. doi: 10.1038/nsmb.1528

Schneider, C., Kudla, G., Wlotzka, W., Tuck, A., and Tollervey, D. (2012). Transcriptome-wide analysis of exosome targets. Mol. Cell. 48, 422-433. doi: 10.1016/j.molcel.2012.08.013

Schneider, C., Leung, E., Brown, J., and Tollervey, D. (2009). The N-terminal PIN domain of the exosome subunit Rrp44 harbors endonuclease activity and 
tethers Rrp44 to the yeast core exosome. Nucleic Acids Res. 37, 1127-1140. doi: 10.1093/nar/gkn1020

Staals, R. H., Bronkhorst, A. W., Schilders, G., Slomovic, S., Schuster, G., Heck, A. J., et al. (2010). Dis3-like 1: a novel exoribonuclease associated with the human exosome. EMBO J. 29, 2358-2367. doi: 10.1038/emboj.20 10.122

Tarasov, A., Vilella, A. J., Cuppen, E., Nijman, I. J., and Prins, P. (2015). Sambamba: fast processing of NGS alignment formats. Bioinformatics 31, 2032-2034. doi: 10.1093/bioinformatics/btv098

Tomecki, R., Kristiansen, M. S., Lykke-Andersen, S., Chlebowski, A., Larsen, K. M., Szczesny, R. J., et al. (2010). The human core exosome interacts with differentially localized processive RNases: hDIS3 and hDIS3L. EMBO J. 29, 2342-2357. doi: 10.1038/emboj.2010.121

Ule, J., Jensen, K., Mele, A., and Darnell, R. B. (2005). CLIP: a method for identifying protein-RNA interaction sites in living cells. Methods 37, 376-386. doi: 10.1016/j.ymeth.2005.07.018

Ule, J., Jensen, K. B., Ruggiu, M., Mele, A., Ule, A., and Darnell, R. B. (2003). CLIP identifies nova-regulated RNA networks in the brain. Science 302, 1212-1215. doi: $10.1126 /$ science. 1090095
Vembar, S. S., Droll, D., and Scherf, A. (2016). Translational regulation in blood stages of the malaria parasite Plasmodium spp.: systems-wide studies pave the way. Wiley Interdiscip. Rev. RNA 7, 772-792. doi: 10.1002/wrna.1365

World Health Organization [WHO], (2018). World Malaria Report. Geneva: WHO Press.

Zhang, Q., Siegel, T. N., Martins, R. M., Wang, F., Cao, J., Gao, Q., et al. (2014). Exonuclease-mediated degradation of nascent RNA silences genes linked to severe malaria. Nature 513, 431-435. doi: 10.1038/nature13468

Conflict of Interest: The authors declare that the research was conducted in the absence of any commercial or financial relationships that could be construed as a potential conflict of interest.

Copyright (c) 2019 Liu, Lu, Fan, He, Shen, Jiang and Zhang. This is an open-access article distributed under the terms of the Creative Commons Attribution License (CC BY). The use, distribution or reproduction in other forums is permitted, provided the original author(s) and the copyright owner(s) are credited and that the original publication in this journal is cited, in accordance with accepted academic practice. No use, distribution or reproduction is permitted which does not comply with these terms. 